\title{
Additional applications of approved radiopharmaceuticals for nuclear cardiology
}

\author{
H. William Strauss • Josef J. Fox
}

Received: 30 August 2013/Accepted: 8 November 2013/Published online: 17 December 2013

(C) Italian Association of Nuclear Medicine and Molecular Imaging 2013

\begin{abstract}
Radionuclide studies of the heart and blood vessels provide information for patient management as well as an understanding of patient-specific pathophysiology. The information gleaned from these studies may be enhanced by using more than a single tracer or by making multiple measurements with a single radiopharmaceutical (similar to measuring thallium redistribution). After more than 40 years of clinical use (Valenta et al., Curr Cardiol Rep 15:344, 2013), the examination with the most clinical value continues to be myocardial perfusion imaging for detection of myocardial ischemia and scar. New techniques, however, are available to expand the application of approved radiopharmaceuticals in patients with heart disease. This manuscript will present recent developments in two areas: (1) Evaluation of mitochondrial function or sympathetic innervation in patients with heart failure (HF). In this case, radionuclide techniques provide data that adds significant predictive value to functional parameters, such as left ventricular ejection fraction, to identify HF patients at high risk of clinical events. (2) Direct evaluation of focal vascular inflammation, microcalcification and calcification in patients with dyslipidemia. Localization and characterization of the intensity of inflammation, or early phases of calcification in atheroma, may permit more personalized therapy than can be obtained from traditional measurements of lipid levels in patients with dyslipidemia. Ultimately, characterizing atheroma, especially in the coronary and carotid arteries, may be the
\end{abstract}

Color figures online at http://link.springer.com/article/10.1007/ s40336-013-0038-7.

H. W. Strauss $(\square)$ J J. J. Fox

Section of Molecular Imaging and Therapy,

Department of Radiology, Memorial Sloan Kettering

Cancer Center, New York, NY, USA

e-mail: straussh@mskcc.org imaging approach of choice to permit effective pharmacologic therapy geared at reducing the incidence and severity of clinical events.

Keywords Heart failure - MIBI washout .

Mitochondrial membrane potential $\cdot \mathrm{mIBG} \cdot$ Vascular inflammation · Vascular calcification

\section{Introduction}

The demonstration of myocardial ischemia in patients with minimal or atypical symptoms and minimal changes on their exercise electrocardiogram (ECG) often results in treatment with medical or revascularization therapy. Adding information on absolute perfusion reserve further increases the value of exercise ECG [1]. Treating these patients before they experience a major clinical event has contributed to the remarkable reduction, in recent years, in the incidence of acute myocardial infarction, while the clinical emphasis on immediate reperfusion has reduced the extent of myocardial scar when an infarct does occur. For example, Kaiser Permanente of Northern California reported a decline in the incidence of ST elevation acute myocardial infarction (STEMI) from 133 cases/100,000 person years in 2003 to 50 cases $/ 100,000$ person years in 2013 [2].

In spite of the decrease in STEMI, there has been an increase in the number of patients with heart failure (HF). The American Heart Association (AHA) estimated that there were 5.1 million people with HF in the USA in 2006 [2]. By 2010, the AHA estimated that the number of HF patients had increased by $20 \%$, to $\sim 6$ million adults [3]. The prevalence of HF in developed countries is $1-2 \%$ of the population, with an incidence of $5-10 / 1,000$ persons per year [4,5]. The rising incidence of $\mathrm{HF}$ represents a new 
challenge to cardiology and an opportunity for cardiovascular nuclear medicine to develop radionuclide techniques for the objective management of these patients.

\section{Heart failure}

Seventy-five percent of HF cases have antecedent hypertension [2]. At 40 years of age, the lifetime risk of developing HF, for both men and women, is 1 in 5 . At 80 years of age, the remaining lifetime risk of developing HF is still $20 \%$ for men and women, even in the face of a much shorter life expectancy. "The lifetime risk of HF occurring without antecedent MI is 1 in 9 for men and 1 in 6 for women." Although the majority of HF starts as diastolic dysfunction, due to the prevalence of myocardial hypertrophy secondary to poorly controlled hypertension, it can progress to dilated myopathy, with an associated decrease in systolic performance.

Common therapies for patients with decreased diastolic function include a beta blocker, an angiotensin-converting enzyme inhibitor or angiotensin II receptor blocker, and an aldosterone antagonist [6]. Common therapies for patients with decreased systolic function include diuretics (e.g., furosemide), angiotensin-converting enzyme inhibitors (e.g., captopril, enalapril or lisinopril), and beta blockers without intrinsic sympathetic activity (e.g., carvedilol, metoprolol, or bisoprolol) [7]. Although measurement of left ventricular ejection fraction provides prognostic information, two other radionuclide techniques, namely (1) quantification of mitochondrial function and (2) quantification of sympathetic innervation, can also provide prognostic data.

The following is a brief discussion of these two approaches, used to obtain prognostic information on patients with HF. The first characterizes myocardial mitochondrial function using an existing tracer, ${ }^{99 \mathrm{~m}} \mathrm{Tc}$-sestamibi; the second characterizes the loss of sympathetic innervation with ${ }^{123} \mathrm{I}-\mathrm{mIBG}$.

\section{Mitochondria}

From the Greek: MITOS $=$ THREAD and KHONDRION $=$ GRAIN.

A mitochondrion is defined as: an organelle in the cytoplasm of cells that functions in energy production [8].

Cardiac myocytes have minimal stores of ATP. A single heart beat consumes $\sim 2 \%$ of ATP in the myocyte; the total ATP pool is turned over within 1 min [9]. To maintain cardiac function, the myocardial mitochondria have the awesome task of generating $\sim 30 \mathrm{~kg}$ of high-energy phosphate every day [10]. ATP is replenished by mitochondrial catabolism of fatty acids and glucose [9]. Fatty

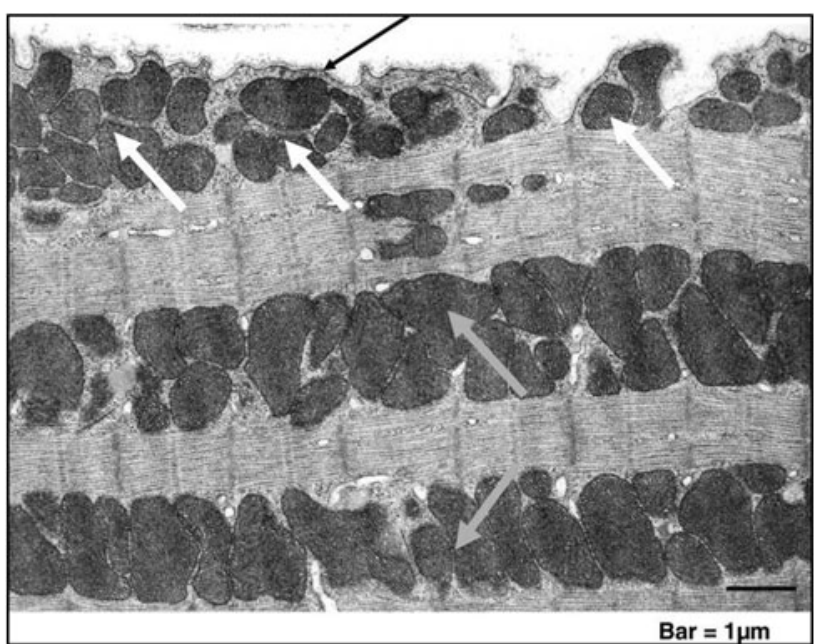

Fig. 1 Electron micrograph of normal myocardium. Sub-sarcolemmal mitochondria (white arrows) located beneath the sarcolemma (black arrow), and longitudinal rows of mitochondria located within the contractile apparatus. From [14]; reprinted with permission

acid is transported into the myocyte via fatty acid transporters. In the cell, coenzyme A is added to the fatty acid, transforming it to acyl-carnitine, which enters mitochondria to produce ATP via beta oxidation. A physiological increase in myocardial work increases fatty acid oxidation to meet the increased demand for ATP. Glucose enters the cell by glucose transporter-enhanced facilitated diffusion. Glucose is phosphorylated to glucose-6-phosphate and undergoes anaerobic glycolysis in the cytoplasm to produce pyruvate. Pyruvate enters mitochondria, where it is converted to acetyl CoA and enters the Krebs cycle to produce ATP. Glucose utilization and fatty acid utilization are reciprocally self-regulating as described by Randle [11] (the Randle cycle). In HF, there is a $30 \%$ reduction in ATP and an even greater reduction in phosphocreatine [12]. The reduction in ATP is associated with decreased mitochondrial membrane potential [13], decreased mitochondrial mass, smaller mitochondrial size, and decreased mitochondrial functional capacity [14].

Electron microscopy of normal cardiac tissue shows mitochondria arranged in two ways in two different locations: (1) in clusters beneath the sarcolemma (subsarcolemmal mitochondria) and (2) in longitudinal rows within the contractile apparatus (interfibrillar mitochondria) (Fig. 1). These two groups of mitochondria appear to have different purposes in the myocyte, with different rates of protein synthesis and membrane turnover [15]. These two locations may be necessary to move ATP from the production sites in mitochondria to sites of utilization [16].

Endocardial biopsies from 48 patients with hypertrophic or dilated cardiomyopathy showed 'pronounced' variation in mitochondrial shape and size; cristae were condensed, 
and in giant mitochondria the cristae were coarse and haphazardly arranged [17]. Mitochondrial changes were present in $100 \%$ of the biopsy specimens. Schaper and colleagues found similar changes in the histopathology of explanted myopathic hearts [18]:

“...mitochondria occurred in large clusters in cytoplasm free of myofibrils, and they varied in size and shape from very small to very large. [There was a] lack of myofibrils in many cellular areas, in the center and in the periphery of a myocyte. Next to hypertrophied cells, other cells were atrophic."

In parallel with the morphometric mitochondrial changes, metabolic remodeling occurs; this is associated with reduced production of ATP [19] and a reduced mitochondrial transmembrane potential $\left(\mathrm{TMP}_{\mathrm{m}}\right)$ [20].

${ }^{99 m}$ Tc-sestamibi (MIBI), a lipophilic tracer with a charge of +1 , is retained in mitochondria [21]. The positively charged fat-soluble tracer molecule traverses the myocardial sarcolemma; in the cell the molecule traverses the cytoplasm to localize in the most electronegative organelle, the mitochondrion. Once in this organelle, the tracer is retained due to the net negative charge. The electronegative charge of myocardial mitochondria is 50-100\% more electronegative than that of the sarcolemmal membrane. Reducing the charge of the mitochondria results in loss of MIBI from the cell. Studies of MIBI retention in cell culture demonstrated that loss of mitochondrial membrane potential resulted in the loss of MIBI from the myocyte $[22,23]$. On the basis of the documented changes in the appearance of mitochondria, and the low levels of ATP in patients with HF, different investigators tested the relationship of myocardial MIBI washout with mechanical dysfunction and prognosis in patients with HF.

In 61 patients with non-ischemic congestive HF, Matsuo et al. [24] found a positive correlation between myocardial MIBI washout rate (WR), increasing NYHA class, and concentration of circulating B-type natruretic peptide, while the WR was inversely correlated with LVEF. Over a mean follow-up of 12 months (range 1-19 months), patients with a WR $\geq 28 \%$ had more events than subjects with a lower WR. Isobe et al. [25] studied 24 patients with hypertrophic myopathy. The investigators recorded planar and SPECT MIBI images following injection at rest. The heart-to-mediastinal ratio (HMR) was calculated from planar images recorded at $40 \mathrm{~min}$ and at $4 \mathrm{~h}$. The investigators found increased MIBI loss from the myocardium in patients with elevated left ventricular end-diastolic pressure and prolonged half time of relaxation. In 17 patients with dilated cardiomyopathy (LVEF $29 \%$ ), Shiroodi and colleagues [26] measured MIBI washout and compared the results to six normal subjects (LVEF $66 \%$ ). Myocardial clearance was measured as the change in HMR at $30 \mathrm{~min}$ and $3.5 \mathrm{~h}$ after injection. Cardiomyopathy patients had a WR of 29 vs $14 \%$ for controls. WR increased from $23 \%$ in patients with NYHA class I to $30 \%$ in class II, $33 \%$ in class III and $38 \%$ in class IV. WR negatively correlated with LVEF $\left(r^{2}=0.679\right)$.

Hayashi and colleagues [27] added significant information to these observations. These investigators studied 20 patients with dilated cardiomyopathy (NYHA class $\mathrm{I}=8$, class $\mathrm{II}=10$ and class $\mathrm{III}=2$; mean $\mathrm{LVEF}=33 \%$ ) divided into two groups on the basis of WR. The group with the higher WR showed a reduction in mRNAs for mitochondrial electron transport-related enzymes on reverse-transcriptase polymerase chain reaction (rtPCR) analysis and more extensive mitochondrial damage on electron microscopy.

In spite of minor differences in technique, Matsuo, Isobe, Shiroodi and Hayashi found a decrease in myocardial MIBI retention in myopathy patients compared to controls. These data suggest that adding a delayed planar image to a myocardial perfusion scan can provide information about mitochondrial status based on the MIBI WR.

As stated by Venturer-Clapier and colleagues: "Despite the diversity of origin and of clinical manifestation of HF, defects in energy metabolism are increasingly considered as an important determinant in the progression of the disease" [28].

\section{Sympathetic innervation}

In patients with HF, cardiac sympathetic neurons show both decreased synthesis of catecholamines [29] and decreased presynaptic norepinephrine reuptake and postsynaptic transport [30]. Using radioiodinated $\mathrm{mIBG}$ as a surrogate for norepinephrine, laboratory studies demonstrated a more rapid efflux of mIBG from the myocardium and human studies revealed less retention of $\mathrm{mIBG}$ in the myocardium, which resulted in a lower heart (myocardial) to HMR in patients with HF [30]. Therapy with angiotensin-converting enzyme inhibitors such as enalapril [31] improved myocardial uptake and retention of $\mathrm{mIBG}$ in patients with HF. The multicenter ADMIRE trial enrolled 961 patients with NYHA class II/III HF and LVEF $\leq 35 \%$ [32]. All subjects were imaged with planar and SPECT mIBG and followed for at least 2 years for progression of $\mathrm{CHF}$, life-threatening arrhythmia, or death. A planar image recorded $\sim 4 \mathrm{~h}$ after injection was used to calculate HMR. This simple measurement provided a reproducible, robust prognostic indicator. The 2-year event rate was $15 \%$ if the HMR was $\geq 1.60$; however, if the HMR was $\leq 1.60$ the event rate increased to $37 \%$. 
Although it is clear that mIBG imaging and calculation of the HMR offers prognostic information in patients with HF, Carrio et al. [30] state:

"From a medical and economic point of view, it is easier to treat all patients with moderately priced drugs that have been shown to benefit a large majority than to attempt to individualize treatment based upon a physiological assessment that might produce equivocal results in a subset of patients."

They suggest, however, that mIBG imaging may be useful in the selection of patients for expensive device therapy such as resynchronization therapy or treatment with implantable defibrillators.

It is unclear at this time which is the more robust prognostic indicator in HF patients: MIBI washout or mIBG HMR. A direct comparison of the two techniques could provide this data.

\section{Vascular inflammation and calcification}

Measuring the degree of inflammation or the formation of new calcification in an atheroma may be useful to define whether the lesion is at risk of causing a clinical event. In 1997, Vallabhajosula and Fuster [33] demonstrated that ${ }^{18}$ F-fluorodeoxyglucose (FDG) could be used to detect vascular inflammation in experimental animals. Five years later, Rudd and colleagues [34] described the findings of FDG PET images of the neck, fused with separate CT studies (to localize the carotid arteries) in eight subjects with carotid atherosclerosis and recent TIA (carotid stenosis $>70 \%$ narrowing). All eight subjects were treated with carotid endarterectomy. Six of the eight patients had asymptomatic lesions contralaterally. There was significantly more FDG in the symptomatic than in the asymptomatic vessel. At the time of endarterectomy, specimens from three patients were incubated with tritiated deoxyglucose for autoradiography. The autoradiographs demonstrated tracer uptake in macrophage-rich lesions.

Since this seminal report, many investigators have validated the ability of FDG vascular imaging to demonstrate focal arterial inflammation in atheroma (summarized by Rudd et al. [35]). To determine whether therapy with simvastatin or dietary management over a period of 3 months reduced FDG uptake in the carotid arteries, Tahara and colleagues [36] randomized 43 patients who demonstrated FDG uptake in their thoracic aorta and/or carotid arteries at the time of baseline FDG PET/CT scan performed for cancer screening. The subjects were divided into two groups: 22 treated with dietary management and 21 treated with simvastatin. Following 3 months of treatment, the patients were reimaged with FDG. The simvastatin group showed a striking reduction in FDG uptake in the carotid arteries, while the diet-treated group did not show a significant change in carotid FDG uptake.

Tawakol et al. [37] correlated carotid FDG uptake on PET/CT scans with CD68 immunostaining of macrophages in carotid endarterectomy specimens, confirming the relationship of FDG uptake with the presence of inflammatory cells in the plaque. FDG vascular uptake in carotid, iliac, and femoral lesions was found to be stable over an interval of 2 weeks [38]. Although this short-term stability is important, the stability of FDG vascular uptake over longer intervals, such as months to years, is likely more critical, since it is the persistence and severity of inflammation that appears to correlate with thin-cap fibroatheroma and clinical events.
Fig. 2 Proposed schematic representation of inflammatory and calcification activity in an atheroma. From [48]; reprinted with permission (color figure online)
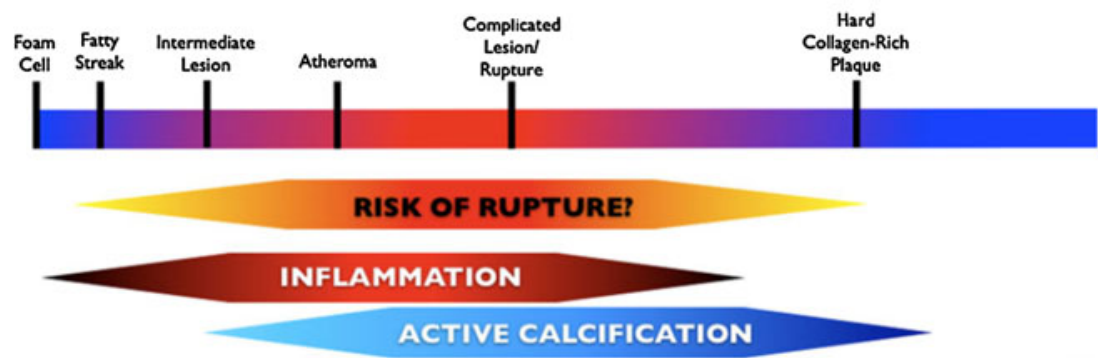

STABLE CALCIUM TURNOVER

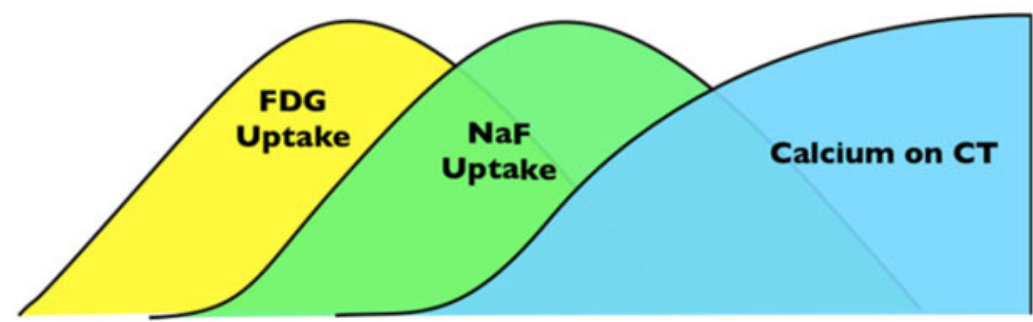


Ben Haim et al. [39] reviewed serial FDG PET/CT studies for vascular FDG uptake and vascular calcification in a series of cancer patients followed with FDG PET/CT. Over an 8- to 26-month interval, she observed changes in the intensity or location of FDG focal uptake in about $48 \%$ of FDG-positive sites. In a similar group of patients undergoing surveillance FDG PET/CT imaging for cancer, Meirelles [40] reported changes in FDG uptake in the thoracic aorta in $55 \%$ of patients over an interval of 21 days to 3 years. These reports suggest that focal vascular inflammation is a waxing and waning phenomenon, with changes occurring at varying intervals. A minority of lesions demonstrated both FDG uptake and calcification. Densely calcified lesions in the vasculature $(>130 \mathrm{HU}$ on $\mathrm{CT}$ ), on the other hand, were relatively stable.

Vascular calcification is a dynamic process, involving inflammation and activation of bone formation pathways [41]. Osteoblasts derived from mesenchymal precursors are responsible for bone formation, while osteoclasts mediate the opposing process of bone resorption [42]. Crystal size is an important parameter, since small crystals of hydroxyapatite are proinflammatory [43], while large dense regions of vascular calcification appear to be more stable.

In contrast to the dense lesions identified on CT, localization of fluoride ions may identify proinflammatory lesions. In the absence of osteoclastic activity, some components of an inflammatory lesion, such as the persistence of apoptotic bodies (which occur in advanced atheroma due to lack of phagocytosis of apoptotic macrophages) serve as a nidus for calcification [44]. This amorphous crystalline structure has a surface composed of many different ions including monovalent, divalent, and trivalent species of both positive and negative charge. ${ }^{18} \mathrm{~F}$-fluoride ions exchange with hydroxyl ions ( $\mathrm{OH}-)$. Because of the small size of the individual crystals, an enormous surface area is exposed [45] (Fig. 2). A major problem with the use of FDG or ${ }^{18} \mathrm{~F}$-fluoride PET/CT to identify vascular inflammation is the limited resolution of the scanner. The $3-5 \mathrm{~mm}$ in plane resolution often limits the ability to define the intense small regions of focal inflammation that may be present in symptomatic lesions. A study by Masteling and colleagues [46] describes this problem very well. These investigators incubated 17 fresh carotid endarterectomy specimens with FDG, imaged the specimens with microPET, and correlated the FDG uptake with the focal presence of macrophages (identified by CD68 immunostaining). The investigators observed a striking topographic correlation between the sites of uptake and the location of macrophages, which was not resolved in vivo on scans performed using standard commercial PET/CT scanners, but was seen on the microPET images of endarterectomy specimens. It is likely that $3 \mathrm{~mm}$ spatial resolution would also limit the ability to identify microcalcifications with ${ }^{18} \mathrm{~F}$-fluoride imaging. The histopathology of atheroma suggests that small lesions with high focal FDG or ${ }^{18} \mathrm{~F}$-fluoride uptake are the most vulnerable. When these lesions are imaged reliably, vascular PET imaging will be ready for routine clinical use.

\section{Summary}

The radionuclide armamentarium for imaging of the cardiovascular system is expanding. This manuscript focused on the development of new uses of existing, commercially available radiopharmaceuticals. Applications in patients with HF and novel approaches to detect inflammation and calcification in atheroma suggest that the field is evolving to meet the growing demands of personalized medicine. A review of the literature suggests that new markers of sympathetic function, inflammation, apoptosis, stem cell migration and survival, and perfusion [47] will ensure a scintillating future for this discipline.

Conflict of interest Neither Dr Strauss nor Dr Fox has a conflict of interest regarding the material presented in this manuscript.

Ethical statement This article does not contain any studies with human or animal subjects performed by either of the authors.

\section{References}

1. Valenta I, Dilsizian V, Quercioli A, Ruddy TD, Scindler TH (2013) Quantitative PET/CT measures myocardial flow reserve and atherosclerosis for cardiac risk assessment and predicting adverse patient outcomes. Curr Cardiol Rep 15:344

2. Go AS, Mozaffarian D, Roger VL, Benjamin EJ, Berry JD, Borden WB, Bravata DM, Dai S, Ford ES et al (2013) Heart disease and stroke statistics-2013 update: a report from the American Heart Association. Circulation 127:e6-e245

3. Roger VL, Go AS, Lloyd-Jones DM, Benjamin EJ et al (2012) Heart disease and stroke statistics. Circulation 125:e2-e220

4. McMurray JJ, Adamopoulos S, Anker SD et al (2012) ESC Committee for Practice Guidelines ESC guidelines for the diagnosis and treatment of acute and chronic heart failure 2012: the task force for the diagnosis and treatment of acute and chronic heart failure 2012 of the European Society of Cardiology. Developed in collaboration with the Heart Failure Association (HFA) of the ESC. Eur J Heart Fail 14:803-869

5. Mosterd A, Hoes AW (2007) Clinical epidemiology of heart failure. Heart 93:1137-1146

6. Colucci WS Overview of heart failure due to systolic dysfunction. In UpToDate updated Jun 7, 2013 Topic 3508 Version 20.0

7. Zaret BL, Strauss HW, Martin ND, Wells HP Jr, Flamm MD Jr (1973) Noninvasive regional myocardial perfusion with radioactive potassium. Study of patients at rest, with exercise and during angina pectoris. N Engl J Med 288:809-812

8. Dictionary.reference.com/browse/mitochondria

9. Nickel A, Loffler J, Maack C (2013) Myocardial energetics in heart failure. Basic Res Cardiol 108:358-378

10. Ashrafian H, Frenneaux MP (2007) Metabolic modulation in heart failure: the coming of age. Cardiovasc Drugs Ther 21:5-7 
11. Randle PJ, Garland PB, Hales CN, Newsholme EA (1963) The glucose fatty-acid cycle. Its role in insulin sensitivity and the metabolic disturbances of diabetes mellitus. Lancet 1:785-789

12. Ingwall JS (2009) Energy metabolism in heart failure and remodelling. Cardiovasc Res 81:412-419

13. Kumar V, Abbas AK, Fausto N (2005) Cell adaptions, cell injury and cell death. Chapter 1. In: Robbins J, Cotran S (eds) Pathologic basis of disease, 7th edn. Elsevier Saunders, Philadelphia, pp 3-46

14. Rosca MG, Hoppel CL (2010) Mitochondria in heart failure. Cardiovasc Res 88:40-50

15. Kasumov T, Dabkowski ER, Shekar KC, Li L, Ribeiro RF Jr, Walsh K, Previs SF, Sadygov RG, Willard B, Stanley WC (2013) Assessment of cardiac proteome dynamics with heavy water: slower protein synthesis rates in interfibrillar than subsarcolemmal mitochondria. Am J Physiol Heart Circ Physiol 304:H1201$\mathrm{H} 1214$

16. Ventura-Clapier Garnier A, Veksler V (2003) Energy metabolism in heart failure. J Physiol 555:1-13

17. Baandrup U, Florio RA, Roters F, Olsen EG (1981) Electron microscopic investigation of endomyocardial biopsy samples in hypertrophy and cardiomyopathy. Circulation 63:1289-1298

18. Schaper J, Froede R, Hein S, Buck A, Hashizume H, Speiser B, Friedl A, Bleese N (1991) Impairment of the myocardial ultrastructure and changes of the cytoskeleton in dilated cardiomyopathy. Circulation 83:504-514

19. Ingwall JS (2009) Energy metabolism in heart failure and remodeling. Cardiovasc Res 81:412-419

20. Dedkova EN, Blatter LA (2012) Measuring mitochondrial function in intact cardiac myocytes. J Mol Cell Cardiol 52:48-61

21. Crane P, Laliberté R, Heminway S, Thoolen M, Orlandi C (1993) Effect of mitochondrial viability and metabolism on technetium99m-sestamibi myocardial retention. Eur J Nucl Med 20:20-25

22. Piwnica-Worms D, Kronauge JF, Chiu ML (1990) Uptake and retention of hexakis (2-methoxyisobutyl isonitrile) technetium(I) in cultured chick myocardial cells. Mitochondrial and plasma membrane potential dependence. Circulation 82:18261838

23. Carvalho PA, Chiu ML, Kronauge JF, Kawamura M, Jones AG, Holman BL, Piwnica-Worms D (1992) Subcellular distribution and analysis of technetium-99m-MIBI in isolated perfused rat hearts. J Nucl Med 33:1516-1521

24. Matsuo S, Nakae I, Tsutamoto T, Okamoto N, Horie M (2007) A novel clinical indicator using Tc-99m sestamibi for evaluating cardiac mitochondrial function in patients with cardiomyopathies. J Nucl Cardiol 14:20-25

25. Isobe $S$, Ohshima $S$, Unno K, Izawa H, Kato K, Noda A, Hirashiki A, Murohara T (2010) Relation of ${ }^{99 \mathrm{~m}} \mathrm{Tc}$-sestamibi washout with myocardial properties in patients with hypertrophic cardiomyopathy. J Nucl Cardiol 17:1082-1190

26. Shiroodi MK, Shafiei B, Baharfard N, Gheidari ME, Nazari B, Pirayesh E, Kiasat A, Hoseinzadeh S, Hashemi A, Akbarzadeh MA, Javadi H, Nabipour I, Assadi M (2012) ${ }^{99 \mathrm{~m}}$ Tc-MIBI washout as a complementary factor in the evaluation of idiopathic dilated cardiomyopathy (IDCM) using myocardial perfusion imaging. Int J Cardiovasc Imaging 28:211-217

27. Hayashi D, Ohshima S, Isobe S, Cheng XW, Unno K, Funahashi H, Shinoda N, Okumura T, Hirashiki A, Kato K, Murohara T (2013) Increased ${ }^{99 \mathrm{~m}} \mathrm{Tc}$-sestamibi washout reflects impaired myocardial contractile and relaxation reserve during dobutamine stress due to mitochondrial dysfunction in dilated cardiomyopathy patients. J Am Coll Cardiol 61:2007-2017

28. Ventura-Clapier R, Garnier A, Veksler V (2004) Energy metabolism in heart failure. J Physiol 555:1-13

29. Rabinovitch MA, Rose CP, Rouleau JL, Chartrand C, Wieland DM, Lepanto L, Legault F, Suissa S, Rosenthall L, Burgess JH
(1987) Metaiodobenzylguanidine $\left[{ }^{131} \mathrm{I}\right]$ scintigraphy detects impaired myocardial sympathetic neuronal transport function of canine mechanical-overload heart failure. Circ Res 61:797-804

30. Carrio I, Cowie MR, Yamazaki J, Udelson J, Camici PG (2010) Cardiac sympathetic imaging with $\mathrm{mIBG}$ in heart failure. JACC Cardiovasc Imaging 3:92-100

31. Somsen GA, van Vlies B, de Milliano PA, Borm JJ, van Royeb EA, Endert E, Lie KI (1996) Increased myocardial [ $\left.{ }^{123} \mathrm{I}\right]-$ metaiodobenzylguanidine uptake after enalapril treatment in patients with chronic heart failure. Heart 73:218-222

32. Jacobson AF, Senior R, Cerqueira MD, Wong ND, Thomas GS, Lopez VA et al (2010) Myocardial iodine-123 meta-iodobenzylguanidine imaging and cardiac events in heart failure. Results of the prospective ADMIRE-HF (AdreView Myocardial Imaging for Risk Evaluation in Heart Failure) study. JACC 55:2212-2221

33. Vallabhajosula S, Fuster V (1997) Atherosclerosis: imaging techniques and the evolving role of nuclear medicine. J Nucl Med 38:1788-1796

34. Rudd JH, Warburton EA, Fryer TD, Jones HA, Clark JC, Antoun N, Johnström P, Davenport AP, Kirkpatrick PJ, Arch BN, Pickard JD, Weissberg PL (2002) Imaging atherosclerotic plaque inflammation with $\left[{ }^{18} \mathrm{~F}\right]$-fluorodeoxyglucose positron emission tomography. Circulation 105:2708-2711

35. Rudd JH, Narula J, Strauss HW, Virmani R, Machac J, Klimas M, Tahara N, Fuster V, Warburton EA, Fayad ZA, Tawakol AA (2010) Imaging atherosclerotic plaque inflammation by fluorodeoxyglucose with positron emission tomography: ready for prime time? J Am Coll Cardiol 55:2527-2535

36. Tahara $\mathrm{N}$, Kai $\mathrm{H}$, Ishibashi $\mathrm{M}$, Nakaura $\mathrm{H}$, Kaida $\mathrm{H}$, Baba $\mathrm{K}$, Hayabuchi N, Imaizumi T (2006) Simvastatin attenuates plaque inflammation: evaluation by fluorodeoxyglucose positron emission tomography. J Am Coll Cardiol 48:1825-1831

37. Tawakol A, Migrino RQ, Bashian GG, Bedri S, Vermylen D, Cury RC, Yates D, LaMuraglia GM, Furie K, Houser S, Gewirtz H, Muller JE, Brady TJ, Fischman AJ (2006) In vivo ${ }^{18}$ F-fluorodeoxyglucose positron emission tomography imaging provides a noninvasive measure of carotid plaque inflammation in patients. J Am Coll Cardiol 48:1818-1824

38. Rudd JH, Myers KS, Bansilal S, Machac J, Pinto CA, Tong C, Rafique A, Hargeaves R, Farkouh M, Fuster V, Fayad ZA (2008) Atherosclerosis inflammation imaging with ${ }^{18}$ F-FDG PET: carotid, iliac, and femoral uptake reproducibility, quantification methods, and recommendations. J Nucl Med 49:871-878

39. Ben Haim S, Kupzov E, Tamir A, Frenkel A, Israel O (2006) Changing patterns of abnormal vascular wall F-18 fluorodeoxyglucose uptake on follow-up PET/CT studies. J Nucl Cardiol 13:791-800

40. Meirelles GS, Gonen M, Strauss HW (2011) ${ }^{18}$ F-FDG Uptake and calcifications in the thoracic aorta on positron emission tomography/computed tomography examinations: frequency and stability on serial scans. J Thorac Imaging 26:54-62

41. Wahlgren CM, Zheng W, Shaalem W, Tang J, Bassiount HS (2009) Human carotid plaque calcification and vulnerability. Cerebrovasc Dis 27:193-200

42. Bostrom K, Watson KE, Horn S, Wortham C, Herman IM, Demer LL (1993) Bone morphogenetic protein expression in human atherosclerotic lesions. J Clin Invest 91:1800-1809

43. Bostrom K (2005) Proinflammatory vascular calcification. Circ Res 96:1219-1220

44. Sage AP, Tintut Y, Demer LL (2010) Regulatory mechanisms in vascular calcification. Nat Rev Cardiol 7:528-536

45. Blau M, Ganatra R, Bender MA (1972) ${ }^{18} \mathrm{~F}$-fluoride for bone imaging. Semin Nucl Med 2:31-37

46. Masteling MG, Zeebregts CJ, Tio RA, Breek JC, Tietge UJF, de Boer JF, Glaudemans J, Dierckx RAJO, Boersma HH, Slart 
RHJA (2011) High-resolution imaging of human atherosclerotic carotid plaques with micro ${ }^{18} \mathrm{~F}$-FDG PET scanning exploring plaque vulnerability. J Nucl Cardiol 18:1066-1075

47. Majmudar MD, Nahrendorf M (2012) Cardiovascular molecular imaging: the road ahead. J Nucl Med 53:673-676
48. Cocker MS, McArdle B, Spence JD, Lum C, Ammond RR, Ongaro DC, McDonald MA, de Kemp RA, Tardif JS, Beanlands RSB (2012) J Nucl Cardiol 19:1211-1225 